\title{
CORRECTION
}

\section{Correction: Trophoblast-derived CXCL16 induces M2 macrophage polarization that in turn inactivates NK cells at the maternal-fetal interface}

Xiao-Qiu Wang ${ }^{1}$, Wen-Jie Zhou' ${ }^{1}$ Xin-Xin $\mathrm{Hou}^{1}$, Qiang $\mathrm{Fu}^{2}$ and Da-Jin $\mathrm{Li}^{1}$

Cellular \& Molecular Immunology (2019) 16:313; https://doi.org/10.1038/s41423-018-0194-9

Correction to: Cellular \& Molecular Immunology (2018) 15, 1038-1046; https://doi.org/10.1038/s41423-018-0019-x; published online 27 March 2018
In this article, published online 27 March 2018, in the "ACKNOWLEDGEMENTS", it should be supplemented as follows: "This study was funded by a grant from the National Natural Science Foundation of China, number 81490744, awarded to DaJin Li." The authors regret the omission.

\footnotetext{
'Laboratory for Reproductive Immunology, Key Laboratory of Reproduction Regulation of NPFPC, SIPPR, Shanghai Key Laboratory of Female Reproductive Endocrine Related Diseases, Hospital and Institute of Obstetrics and Gynecology, IRD, Fudan University, Shanghai Medical College, Shanghai, China and ${ }^{2}$ College of Basic Medicine, Binzhou Medical University, Yantai, Shandong, China

Correspondence: D.-J. Li (djli@shmu.edu.cn)

These authors contributed equally: Xiao-Qiu Wang, Wen-Jie Zhou.
}

Published online: 22 January 2019 